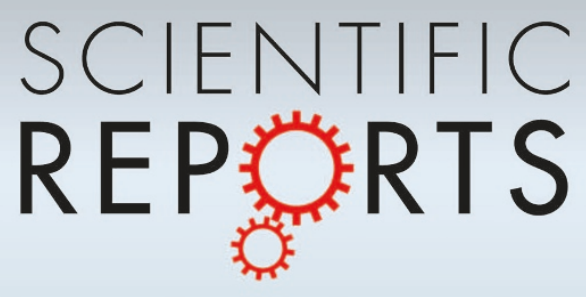

OPEN

SUBJECT AREAS:

ELECTRONIC STRUCTURE SUPERCONDUCTING PROPERTIES AND MATERIALS

APPLIED PHYSICS

SUPERCONDUCTORS

Received

5 August 2013

Accepted

12 November 2013

Published

26 November 2013

Correspondence and requests for materials should be addressed to K.M. (kbmaiti@tifr.res.

in)

\footnotetext{
* Current address: Paul

Scherrer Institut, $\mathrm{CH}$ 5232 Villigen PSI, SWITZERLAND
}

\section{Complex spectral evolution in a BCS superconductor, $\mathrm{ZrB}_{12}$}

\author{
Sangeeta Thakur', Deepnarayan Biswas' ${ }^{1}$ Nishaina Sahadev', P. K. Biswas ${ }^{2 *}$, G. Balakrishnan ${ }^{2}$ \\ \& Kalobaran Maiti'
}

'Department of Condensed Matter Physics and Materials' Science, Tata Institute of Fundamental Research, Colaba, Mumbai - 400 005, India, ${ }^{2}$ Department of Physics, University of Warwick, Coventry, CV4 7AL, UK.

We investigate the electronic structure of a complex conventional superconductor, $\mathrm{ZrB}_{12}$ employing high resolution photoemission spectroscopy and $a b$ initio band structure calculations. The experimental valence band spectra could be described reasonably well within the local density approximation. Energy bands close to the Fermi level possess $t_{2 g}$ symmetry and the Fermi level is found to be in the proximity of quantum fluctuation regime. The spectral lineshape in the high resolution spectra is complex exhibiting signature of a deviation from Fermi liquid behavior. A dip at the Fermi level emerges above the superconducting transition temperature that gradually grows with the decrease in temperature. The spectral simulation of the dip and spectral lineshape based on a phenomenological self energy suggests finite electron pair lifetime and a pseudogap above the superconducting transition temperature.

he relationship between pseudogap phase and superconductivity in high temperature superconductors is an issue of discussion for many years now ${ }^{1-3}$. While one school believes that the pseudogap arises due to some hidden order and/or effects not associated to superconductivity, the other school attributes pseudogap to the electron pair formation above the transition temperature, $T_{c}$ as a precursor to the superconducting gap that leads to superconductivity upon establishment of the coherence among the electron pairs at $T_{c}$. Independent of these differences, it is realized that electron correlation plays a significant role in the occurrence of pseudogap phase in these unconventional superconductors ${ }^{4,5}$. Several questions are being asked on whether the pseudogap phase is specific to unconventional superconductors, electron correlation or low dimensionality is a necessity for such phase etc.

Apart from these issues, the quest of new superconducting compounds resulted into the discovery of superconductivity in cubic hexane, $M_{6}$ and dodecaborides, $M B_{12}(M=\mathrm{Sc}$. Y, Zr, La, Lu, Th) those attracted much attention due to their interesting electronic properties ${ }^{6} . \mathrm{ZrB}_{12}$ is one such compound exhibiting relatively high superconducting transition temperature of $6 \mathrm{~K}$ in the $\mathrm{MB}_{12}$ family ${ }^{6}$. It forms in cubic structure as shown in Fig. 1. In Fig. 1(a), the real lattice positions are shown with the correct bond lengths and atom positions. Although, the structure appears to be complex, it is essentially a rock salt structure constituted by two interpenetrated fcc lattices formed by $\mathrm{Zr}$ and $\mathrm{B}_{12}$ units as demonstrated in Fig. 1(b) by compressing the $\mathrm{B}_{12}$ units for clarity. Various transport and magnetic measurements suggest a Bardeen-Cooper-Schriefer (BCS) type superconductivity in this material, which is termed as conventional superconductivity. Since, $\mathrm{Zr}$ atoms are located within the huge octahedral void space formed by the $\mathrm{B}_{12}$ units, the superconductivity in these materials could conveniently be explained by the electron-phonon coupling mediated phenomena involving primarily low energy phonon modes associated with the vibration of $\mathrm{Zr}$ atoms ${ }^{7}$.

The electronic properties of $\mathrm{ZrB}_{12}$ manifest plethora of conflicts involving the mechanism of the transition. For example, there are conflicts on whether it is a type I or type II superconductor ${ }^{8}$, whether it possesses single gap ${ }^{9}$ or $^{2}$ multiple gaps ${ }^{10}$, etc. Interestingly, Gasparov et al..$^{10}$ found that superfluid density of $\mathrm{ZrB}_{12}$ exhibits unconventional temperature dependence with pronounced shoulder at $T / T_{c} \sim 0.65$. In addition, they find different superconducting gap and transition temperatures for different energy bands, and the values of the order parameter obtained for $p$ and $d$ bands are 2.81 and 6.44, respectively. Detailed magnetic measurements reveal signature of Meissner, mixed and intermediate states at different temperatures and magnetic fields ${ }^{11}$. Some of these variances were attributed to surface-bulk differences in the electronic structure ${ }^{12}$, the superconductivity at the sample surface ${ }^{13-15}$ etc. Evidently, the properties of $\mathrm{ZrB}_{12}$ is complex despite exhibiting signatures of BCS type superconductivity. Here, we probed the evolution of the electronic structure of $\mathrm{ZrB}_{12}$ employing high resolution photoelectron spectroscopy. The experimental results exhibit spectral evolution anomalous to conventional type superconductor and signature of pseudogap prior to the onset of superconductivity. 

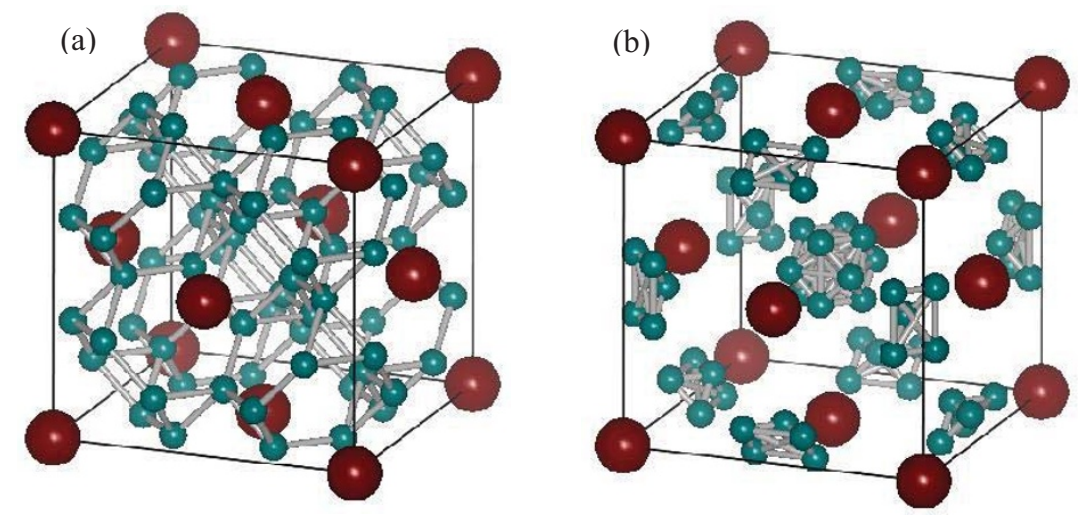

Figure $1 \mid$ (a) Crystal structure of $\mathrm{ZrB}_{12}$. (b) $\mathrm{B}_{12}$ units are contracted to visualize the isolated $\mathrm{B}_{12}$ clusters with better clarity.

\section{Results}

Valence band spectra obtained using $\mathrm{He}$ II and $\mathrm{Al} \mathrm{K} \alpha$ excitation energies are shown in Fig. 2(a) exhibiting three distinct features marked by $\mathrm{A}, \mathrm{B}$ and $\mathrm{C}$ beyond $2 \mathrm{eV}$ binding energies. The intensities between $6-10 \mathrm{eV}$ binding energies represented by $\mathrm{B}$ are prominent in the $\mathrm{He}$ II spectrum, while the relative intensity between $3-4 \mathrm{eV}$ binding energies represented by $\mathrm{A}$ is enhanced in the $x$-ray photoemission (XP) spectrum. Such change in intensity in the angle integrated spectra may be attributed to the matrix elements associated to different constituent states forming the eigenstates of the valence band, which is a sensitive function of the photon energy ${ }^{16}$. Therefore, the photoemission cross section will vary with the photon energy that can be used to identify the orbital character of the energy bands. Considering this feature of the technique, the spectral feature,
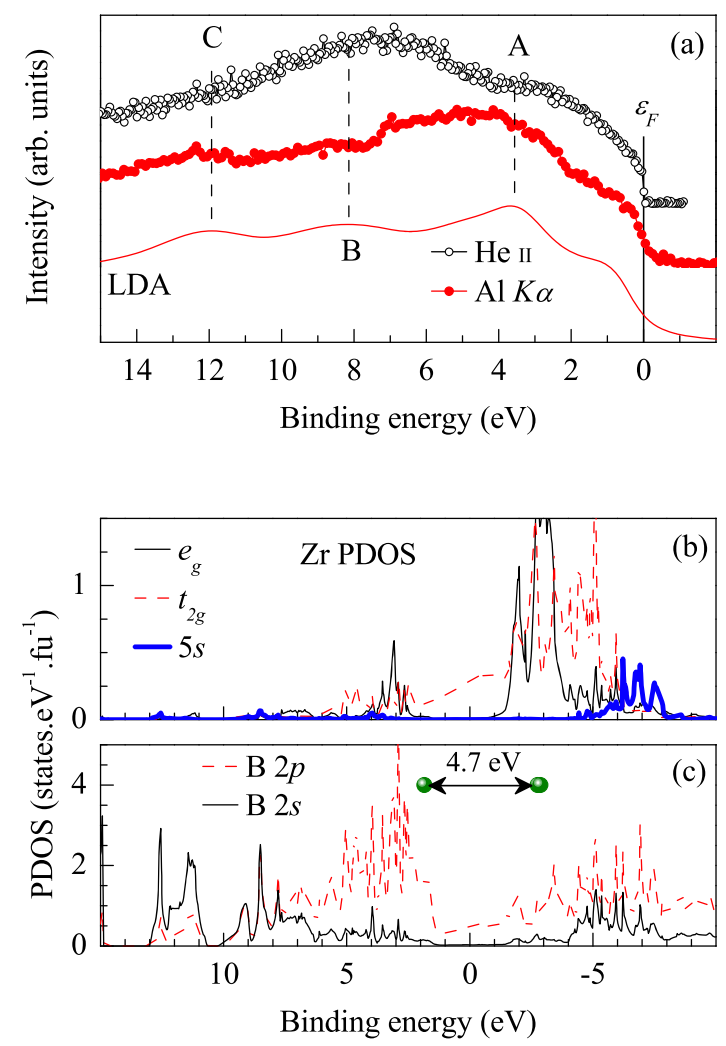

Figure $2 \mid$ (a) XP and He II valence band spectra of $\mathrm{ZrB}_{12}$. The lines show the simulated XP spectrum from the band structure results. Calculated partial density of states of (b) $\mathrm{Zr} 4 d t_{2 g}$ (dashed line), $4 d e_{g}$ (thin solid line) $\& 5 s$ (thick solid line), and (c) B $2 s$ (solid line) and $2 p$ (dashed line) partial density of states.
B can be attributed to dominant B $2 p$ orbital character and the feature A to $\mathrm{Zr} 4 d$ orbital character.

Energy band structure of $\mathrm{ZrB}_{12}$ has been calculated within the local density approximations (LDA). The calculated partial density of states (PDOS) corresponding to $\mathrm{Zr} 4 d \& 5 s$, and B $2 s \& 2 p$ states are shown in Fig. 2(b) and 2(c), respectively. Zr $5 s$ contribution appears in the energy range of 5-7.5 eV above the Fermi level with negligible contributions in the energy window studied here as shown by thick solid line in Fig. 2(b). B $2 s$ contributions appear beyond $6 \mathrm{eV}$ binding energies. Dominant contribution from B $2 p$ PDOS appears between 1.5 to $10 \mathrm{eV}$ binding energies. The center of mass of the B $2 p$ PDOS (considering both occupied and unoccupied parts) appears around $1.9 \mathrm{eV}$ binding energy. The center of mass of the entire $\mathrm{Zr} 4 d$ PDOS appear around $2.8 \mathrm{eV}$ above $\epsilon_{F}$, which is about $4.7 \mathrm{eV}$ above the center of mass of the B $2 p$ PDOS. Thus, an estimate of the charge transfer energy could be about $4.7 \mathrm{eV}$ in this system. The ground state is metallic with flat density of states across $\epsilon_{F}$ arising due to highly dispersive energy bands in this energy range. The density of states at $\epsilon_{F}$ possess close to $2: 1$ intensity ratio of the $\mathrm{B} 2 p$ and $\mathrm{Zr} 4 d$ orbital character.

The electronic states with $t_{2 g}$ and $e_{g}$ symmetries, shown in Fig. 2(b), exhibit almost overlapping center of mass suggesting negligible crystal field splitting (crystal field splitting $\sim 100 \mathrm{meV}$ ). The energy bands are shown in Fig. 3. $e_{g}$ bands are relatively narrow and exhibit a gap at $\epsilon_{F}$. The intensity at $\epsilon_{F}$ essentially have highly dispersive $t_{2 g}$ symmetry.

Using the above band structure results, we calculated the XP spectrum considering the photoemission cross section of the constituent partial density of states as discussed later in the Method section. The calculated spectrum shown by solid line in Fig. 2(a) exhibits an excellent representation of the experimental XP spectrum. It is clear that the feature, $\mathrm{C}$ in the energy range $10-14 \mathrm{eV}$ corresponds to photoemission signal from B $2 s$ levels. The features, A and B arise due to hybridized B $2 p-\mathrm{Zr} 4 d$ states. The relative contribution from $\mathrm{Zr} 4 d$ states is higher for the feature A.

In order to probe the electron correlation induced effect on the electronic structure, we have carried out electron density of states calculation using finite electron correlation among the $\mathrm{Zr} 4 d$ electrons, $U_{d d}$ as well as that among B $2 p$ electrons, $U_{p p}$. In Figs. 4(a) and $4(\mathrm{~b})$, we show the calculated results for $\mathrm{Zr} 4 d$ and B $2 p$ PDOS with $U_{d d}=0$ (solid line) and $6 \mathrm{eV}$ (dashed line) for the $\mathrm{Zr} 4 d$ electrons. Even the large value of $U_{d d}$ of $6 \mathrm{eV}$ does not have significant influence on the occupied part of the electronic structure, while an energy shift is observed in the unoccupied part. Such scenario is not unusual considering the large radial extension of $4 d$ orbitals and poor occupancy ${ }^{17}$. The inclusion of an electron correlation, $U_{p p}$ of upto $4 \mathrm{eV}$ for B $2 p$ states exhibits insignificant change in the results. Considering $\mathrm{Zr}$ being a heavy element, we carried out similar calculations including spin-orbit interactions and did not find significant modification in 
(a) $t_{2 g}$ bands

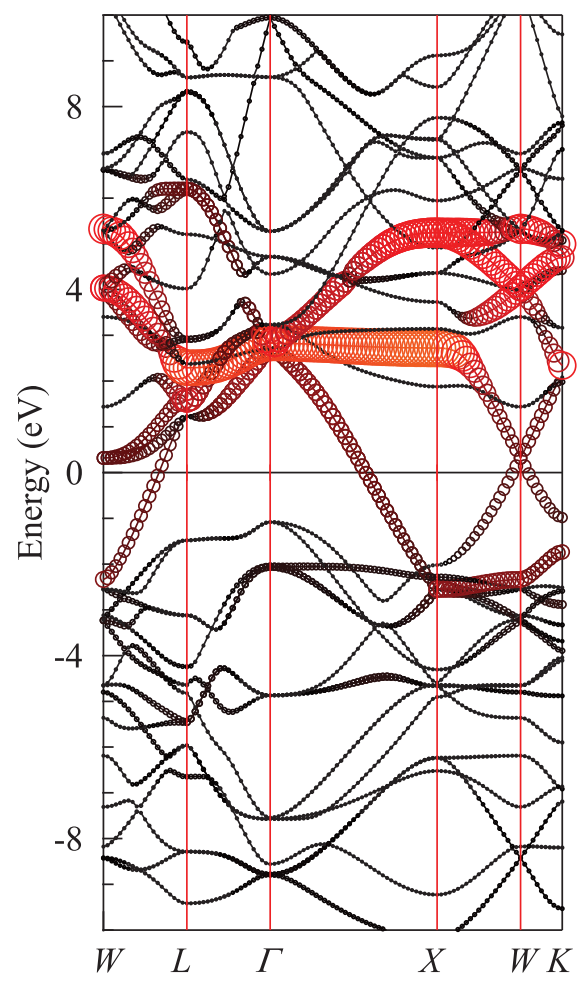

(b) $e_{g}$ bands

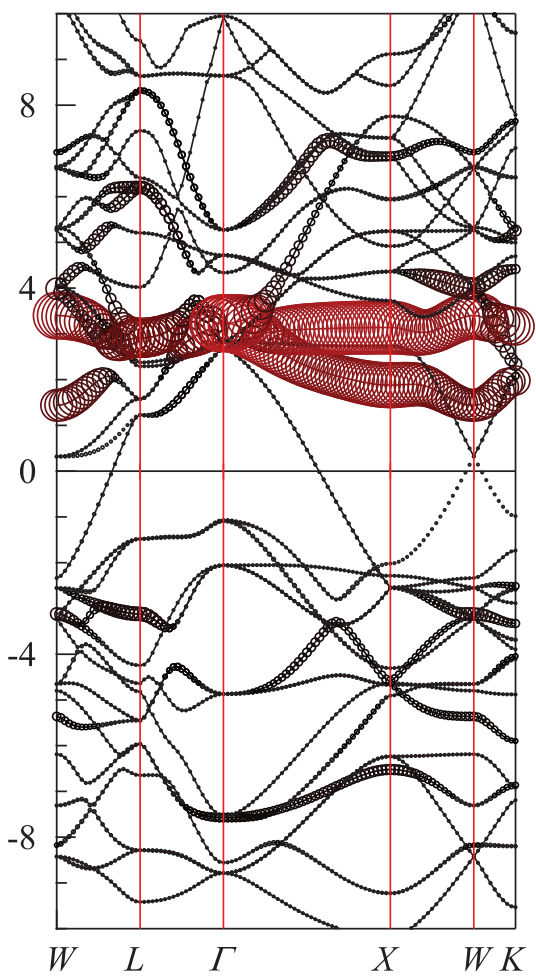

Figure $3 \mid$ Energy band dispersions. Symbol size in the left panel show $t_{2 g}$ contributions and that on the right panel shows $e_{g}$ contributions.
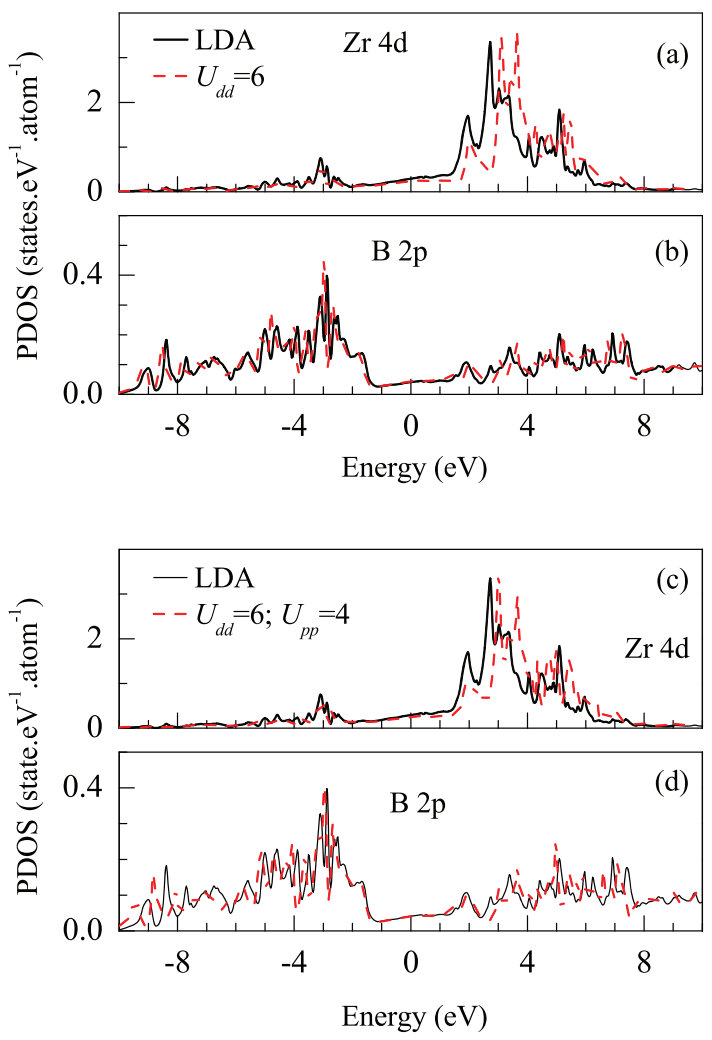

Figure $4 \mid$ (a) Zr $4 d$ and (b) B $2 p$ PDOS obtained from LDA and LDA $+U$ calculations $\left(U_{d d}=6 \mathrm{eV}\right)$. (c) $\mathrm{Zr} 4 d$ and (d) B $2 p$ PDOS obtained for $U_{d d}=$ $6 \mathrm{eV}$ and $U_{p p}=4 \mathrm{eV}$. the results - we have not shown these later results to maintain clarity in the figure. All these theoretical results suggest that consideration of electron correlation within the LSDA $+U$ method has insignificant influence in the occupied part of the electronic structure of this system.

We now investigate the spectral changes near ${ }_{F}$ with high energy resolution in Fig. 5. The spectral density of states (SDOS) are calculated by symmetrizing the experimental spectra; $S D O S=I\left(\epsilon-\epsilon_{F}\right)+$ $I\left(\epsilon_{F}-\epsilon\right)$ and shown in Figs. 5(c) and 5(d). Such an estimation of SDOS is sensitive to the definition of the Fermi level, which is carefully derived at each temperature by the Fermi cutoff in the valence band spectra for silver mounted on the sample holder in electrical connection with the sample and measured using identical experimental conditions. The other effect is the asymmetry across the Fermi level. This can be addressed by obtaining the SDOS by the division of the resolution broadened Fermi-Dirac distribution function. The results are shown in Figs. 5(e) and 5(f) for He II and He I spectra, respectively. Clearly, the SDOS obtained following the later procedure exhibits symmetric SDOS across $\epsilon_{F}$. The $30 \mathrm{~K}$ and $10 \mathrm{~K}$ data exhibit large noise above $\epsilon_{F}$ due to the division of negligibly small intensities by small numbers arising from Fermi-Dirac function at these temperatures. These results demonstrate the reliability of SDOS extraction procedures and also indicate signature of particle-hole symmetry in this system.

The spectral lineshape near the Fermi level often provides important information about the thermodynamic properties of the systems. For example, a lineshape dependence of $\left|\epsilon-\epsilon_{F}\right|^{2}$ corresponds to Fermi liquid behavior ${ }^{18,19}$ - absence of linearity of SDOS with $\left|\epsilon-\epsilon_{F}\right|^{2}$ shown in Fig. 6(a) indicates a deviation from such a behavior in the present case. The decrease in intensity at $\epsilon_{F}$ with the decrease in temperature may arise due to the disorder induced localization of the electronic states. Altshuler \& Aronov showed that the charge 

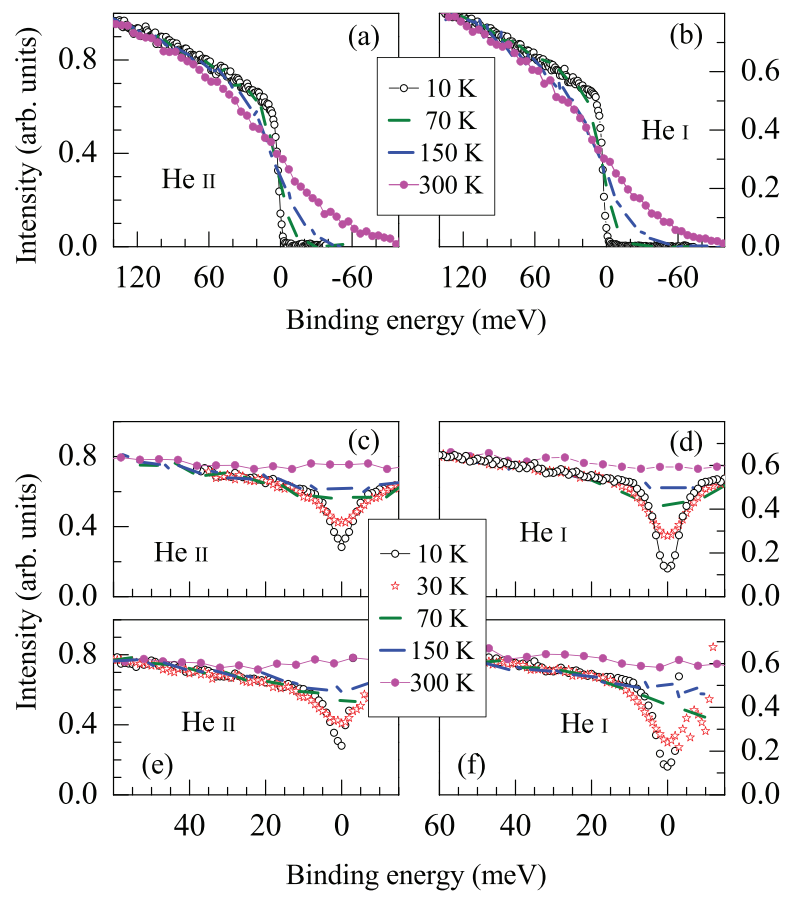

Figure $5 \mid$ High resolution spectra near the Fermi level using (a) He II and (b) He I photon energies. The symmetrized spectral density of states (SDOS) for (c) He II and (d) He I spectra, and SDOS obtained by division of resolution broadened Fermi function for (e) He II and (f) for He I spectra.

disorder in a correlated system leads to $\left|\epsilon-\epsilon_{F}\right|^{0.5}$ dependence of the density of states near Fermi level ${ }^{20}$, which has been proved employing photoemission spectroscopy ${ }^{21,22}$. In the present case, a plot of SDOS with $\left|\epsilon-\epsilon_{F}\right|^{0.5}$ shown in Fig. 6(b) exhibits deviation from linearity.

Interestingly, the curvatures at the exponents of 0.5 and 2 are opposite indicating an intermediate exponent for the present system. The simulation of SDOS as a function of $\left|\left(\epsilon-\epsilon_{f}\right)\right|^{\alpha}$ with $\alpha \approx 0.9$ exhibits linear dependence with energy for all the temperatures studied (see Fig. 6(c)). Such a behavior in a conventional superconductor is curious. Most interestingly, in Fig. 6(c), all the data below $150 \mathrm{~K}$ superimpose exactly and correspond to the same energy scale, while the data at $300 \mathrm{~K}$ exhibit similar energy dependence with a different slope. These results suggest signature of an incipient phase transition at an intermediate temperature.

The SDOS at $\epsilon_{F}$ is intense and flat at $300 \mathrm{~K}$ with large dispersion of the bands and correspond to a good metallic phase consistent with the band structure results. The decrease in temperature introduces a dip at $\epsilon_{F}$. The dip gradually becomes more prominent with the decrease in temperature. The same scenario is observed in both, He I and He II spectra indicating this behavior to be independent of the photon energy used. This dip may be attributed to a pseudogap appearing at low temperatures ${ }^{23}$. Although the probed lowest temperature is slightly above $T_{c}$, the dip grows monotonically with the decrease in temperature - a large suppression of the spectral weight at the Fermi level is observed at $10 \mathrm{~K}$ indicating relation of this dip to the superconducting gap. In order to estimate the energy gap in these spectral functions, we used a phenomenological self-energy, $\Sigma_{k}(\epsilon)$ following the literature ${ }^{24}$.

$$
\Sigma_{k}(\epsilon)=-i \Gamma_{1}+\Delta^{2} /\left[\left(\epsilon+i \Gamma_{0}\right)+\epsilon_{k}\right]
$$

where $\Delta$ represents the gap size. The first term is the energy independent single particle scattering rate and the second term is the BCS self energy. $\Gamma_{0}$ represents the inverse electron pair lifetime. The spectral functions, the imaginary part of the Green's functions, were
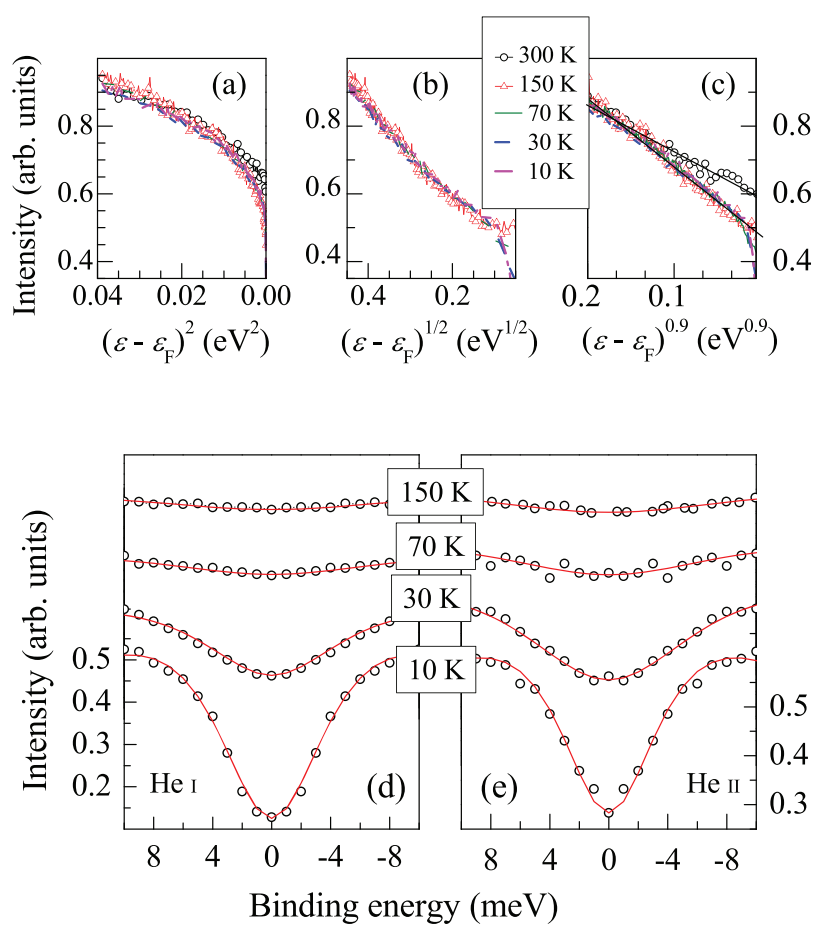

Figure $6 \mid$ High resolution spectral density of states is shown as a function of (a) $\left|\epsilon-\epsilon_{F}\right|^{2}$, (b) $\left|\epsilon-\epsilon_{F}\right|^{1 / 2}$, and (c) $\left|\epsilon-\epsilon_{F}\right|^{0.9}$. Spectral density of states from (d) He I and (e) He II spectra. The lines represent the spectral functions simulated to fit the experimental spectra.

calculated as follows. $A(\epsilon)=(1 / \pi) \operatorname{Im} \Sigma_{k} G_{k}(\epsilon)=(1 / \pi) \Sigma_{k} \Sigma_{k}^{\prime \prime}(\epsilon) /$ $\left[\left(\epsilon-\epsilon_{k}-\Sigma_{k}^{\prime}(\epsilon)\right)^{2}+\left(\Sigma_{k}^{\prime \prime}(\epsilon)\right)^{2}\right]$, where $\Sigma_{k}^{\prime}(\epsilon)$ and $\Sigma_{k}^{\prime \prime}(\epsilon)$ are the real and imaginary part of the self energy. We find that a finite value of $\Gamma_{0}$ is necessary to achieve reasonable representation of the experimental data. The simulated and experimental spectral density of states are shown by superimposing them in Figs. 6(d) and 6(e) for the $\mathrm{He}$ I and He II spectra, respectively. Evidently, the simulated spectra provide a remarkable representation of the experimental spectra.

\section{Discussion}

The experimental and calculated band structure results suggest significant hybridization between $\mathrm{Zr} 4 d$ and $\mathrm{B} 2 p$ states leading to similar energy distribution of the PDOS. Here, the eigenstates are primarily constituted by the linear combination of the $\mathrm{Zr} 4 d$ and B $2 p$ states. The antibonding bands possess large $\mathrm{Zr} 4 d$ orbital character and appear in the unoccupied part of the electronic structure ${ }^{25,26}$. The energy bands below $\epsilon_{F}$ forming the valence band are the bonding eigenstates consisting of dominant $2 p$ orbital character. The covalency between these states could be attributed to the large radial extension of the $4 d$ orbitals overlapping strongly with the neighboring states as also observed in other $4 d$ systems $^{27,28}$. The calculated results within the local density approximation are consistent with the experimental spectra - subtle differences between the experiment and theory could not be captured via inclusion of electron correlation and/or consideration of spin-orbit coupling in these LDA calculations.

The band structure results exhibit two important features - (i) the energy bands with $t_{2 g}$ symmetry are highly dispersive compared to $e_{g}$ bands and (ii) the crystal field splitting is negligible. As shown in Fig. 1, the $\mathrm{B}_{12}$ dodecahedrons form an octahedra around $\mathrm{Zr}$ sites with the center of mass of the $B_{12}$ units at the edge centers of the unit cell. The size of the dodecahedrons are quite large with no boron situating on the unit cell edge. Thus, the overlap of B $2 p$ orbitals with the $t_{2 g}$ 


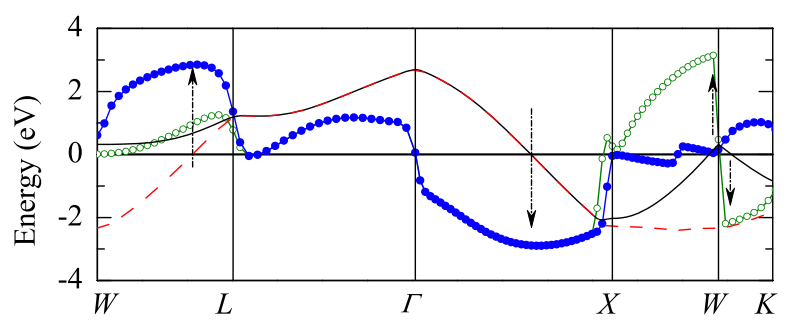

Figure $7 \mid$ Energy band dispersions of the $t_{2 g}$ bands crossing the Fermi level are shown by lines. The symbols represent the first derivative of the same energy bands. The arrows show the Fermi level crossings.

orbitals is significantly enhanced due to the proximity of borons along the face diagonals, while that with the $e_{g}$ orbitals is reduced leading to a comparable crystal field potential on both the orbitals. The larger hybridization with the $t_{2 g}$ orbitals are also manifested by the large dispersion of the $t_{2 g}$ bands spanning across the Fermi level while $e_{g}$ bands are relatively more localized. From these calculations, the effective valency of $\mathrm{Zr}$ was found to vary between $(+2.4)$ to $(+2.5)$ depending on the electron interaction parameters such as correlation strength, spin orbit coupling etc.

The Fermi level is located almost at the inflection points of each of the energy band crossing $\epsilon_{F}$ as shown in Fig. 7. This is verified by investigating the first derivative of the energy bands shown by the symbols in the figure. The arrows in the figure indicate Fermi level crossing and corresponding point in the first derivative curves. Evidently, the band crossing correspond to an extremum in the derivative plot. The inflection point is the point, where the curve changes its curvature that corresponds to a change in the character of the conduction electrons (the Fermi surface) from hole-like to electron-like behavior. This behavior is termed as Lifschitz transition $^{29}$. Thus, this material appear to be lying in the proximity of the Lifschitz transition as also observed in various exotic unconventional superconductors such as Fe-pnictides ${ }^{30}$. Due to the lack of cleavage plane and hardness of the sample, the Fermi surface mapping of this sample could not be carried out to verify this theoretical prediction experimentally. It is well known that the band structure calculations captures the features in the electronic structure well in the weakly correlated systems as also found in the present case suggesting possibility of such interesting phenomena in this system. We hope, future studies would help to enlighten this issue further.

The high resolution spectra close to the Fermi level indeed exhibit deviation from Fermi liquid behavior - a suggestive of quantum instability in this system as expected from the above results. R. Lortz et al. ${ }^{7}$ showed that the resistivity of $\mathrm{ZrB}_{12}$ exhibits linear temperature dependence in a large temperature range of $50 \mathrm{~K}-300 \mathrm{~K}$. The resistivity below $50 \mathrm{~K}$ of the normal phase is complex and does not show $T^{2}$-dependence. Both, the resistivity and specific heat data exhibit signature of anharmonic mode of $\mathrm{Zr}$-vibrations. All these observations indicate complexity of the electronic properties, different from a typical Fermi-liquid system consistent with the photoemission results.

A dip appears at the Fermi level at temperatures much higher than the superconducting transition temperature reminiscent of a pseudogap feature. In order to investigate this further, we study the fitting parameters simulating the spectral functions in Fig. 8. The energy gap, $\Delta$ is found to be about $7.3 \mathrm{meV}$ and remains almost unchanged in the whole temperature range studied. This gap is significantly larger than the value predicted (1-2 meV) considering BCS behavior. The magnitude of $\Gamma_{1}$, which is a measure of single particle scattering rate, is quite large and found to decrease with the decrease in temperature. On the other hand, $\Gamma_{0}$, the inverse pair lifetime is found to be significant and smaller than $2 \Delta$ suggesting proximity to a BCS limit for this system. $\Gamma_{0}$ exhibits temperature dependence quite similar to that of $\Gamma_{1}$. The decrease in $\Gamma_{0}$ manifests gradual

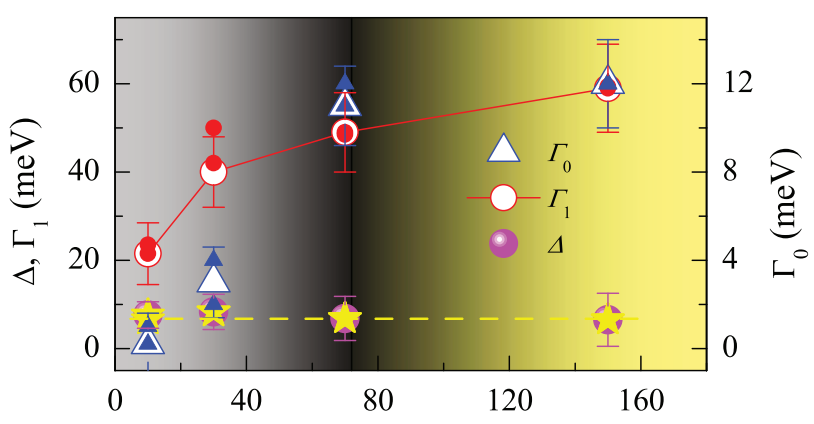

Temperature (K)

Figure $8 \mid$ The gap, $\Delta$, inverse pair lifetime, $\Gamma_{0}$ and single particle scattering rate, $\Gamma_{1}$ as a function of temperature. The open and close symbols correspond to the fitting of $\mathrm{He}$ I and $\mathrm{He}$ II data, respectively.

enhancement of the electron pair lifetime with the decrease in temperature that eventually leads to the superconducting phase upon attaining coherence among the pairs. Thus, the temperature in the vicinity of $70 \mathrm{~K}$ exhibiting the onset of pair formation could be the critical temperature, $T^{*}$ in this system. The observation of the signature of an energy gap and finite electron pair lifetime above $T_{c}$ is quite similar to that observed for under-doped cuprates ${ }^{24}$ and is an indication of precursor effect in this system. Clearly, the electronic structure of this system is complex, appears to be at the crossover of BCS and unconventional behaviors and further studies are required to understand the origin of pseudogap \& unusual spectral lineshape.

In summary, we studied the electronic structure of $\mathrm{ZrB}_{12}$, predicted to be a complex conventional superconductor employing high resolution photoemission spectroscopy and $a b$ initio band structure calculations. The valence band spectra exhibit multiple features with dominant B $2 p$ orbital character close to the Fermi level. The experimental results could be captured reasonably well within the local density approximations. The electronic states close to Fermi level have $t_{2 g}$ symmetry and the filling of the bands is in the proximity of Lifschitz transition. The spectral lineshape near the Fermi level in the high resolution spectra exhibit deviation from typical Fermi liquid behavior. A dip at the Fermi level emerges above the superconducting transition temperature and gradually becomes prominent at lower temperatures. The analysis of the spectral functions within the phenomenological descriptions suggests finite electron pair lifetime above $T_{c}$ as a signature of a precursor effect to the superconducting transition in this system.

\section{Methods}

Sample preparation and characterization. Single crystals of $\mathrm{ZrB}_{12}$ were grown by floating zone technique in a Crystal Systems Incorporated (CSI) four-mirror infrared image furnace in flowing high purity Ar gas at a pressure of $2 \mathrm{bar}^{31}$. The quality of the crystal was confirmed and orientation was determined from $x$-ray Laue - diffraction images. The magnetization measurements using a Qunatum Design MPMS magnetometer show a sharp superconducting transition temperature, $T_{c}$ of $6.1 \mathrm{~K}^{11}$.

Photoemission measurements. The photoemission measurements were performed using a Gammadata Scienta R4000 WAL electron analyzer and monochromatic laboratory photon sources. $X$-ray photoemission (XP) and ultraviolet photoemission (UP) spectroscopic measurements were carried out using $\mathrm{Al} \mathrm{K} \alpha(1486.6 \mathrm{eV})$, He II $(40.8 \mathrm{eV})$ and $\mathrm{He} \mathrm{I}(21.2 \mathrm{eV})$ photon energies with the energy resolution set to $400 \mathrm{meV}, 4 \mathrm{meV}$ and $2 \mathrm{meV}$, respectively. The melt grown sample was very hard and possesses no cleavage plane. Therefore, the sample surface was cleaned using two independent methods; top-post fracturing and scraping by a small grain diamond file at a vacuum better than $3 \times 10^{-11}$ torr. Both the procedures results to similar spectra with no trace of impurity related signal in the photoemission measurements. The measurements were carried out in analyzer transmission mode at an acceptance angle of $30^{\circ}$ and reproducibility of all the spectra was ensured after each surface cleaning cycle. The temperature variation down to $10 \mathrm{~K}$ was achieved by an open cycle $\mathrm{He}$ cryostat from Advanced Research systems, USA.

Band structure calculations. The energy band structure of $\mathrm{ZrB}_{12}$ was calculated using full potential linearized augmented plane wave method within the local density 
approximation (LDA) using Wien2k software ${ }^{32}$. The energy convergence was achieved using $512 k$-points within the first Brillouin zone. The lattice constant of $7.4075 \AA$ was used considering the unit cell shown in Fig. $1^{33}$. In order to introduce electron-electron Coulomb repulsion into the calculation, we have employed LDA + $U$ method with an effective electron interaction strength, $U_{\text {eff }}(=U-J ; J=$ Hund's exchange integral) setting $J=0$ following Anisimov et al ${ }^{34}$. Consideration of finite $J$ did not have significant influence on the results. We have carried out the calculations for various values of electron correlation upto $6 \mathrm{eV}$ for $\mathrm{Zr} 4 d$ electrons and $4 \mathrm{eV}$ for $\mathrm{B}$ $2 p$ electrons. Spin-orbit interactions among the $\mathrm{Zr} 4 d$ electrons are also considered for the calculations.

To calculate the $x$-ray photoemission spectrum, we have multiplied the partial density of states obtained by the band structure calculations by the corresponding photoemission cross sections for $\mathrm{Al} \mathrm{K \alpha}$ energy. These cross-section weighted PDOS is convoluted by two Lorentzians with the energy dependent full width at half maxima (FWHM) for the hole \& electron lifetime broadenings and a Gaussian with FWHM representing the resolution broadening. The sum of these spectral functions provides the representation of the experimental valence band spectrum.

1. Vishik, I. M. et al. ARPES studies of cuprate Fermiology: superconductivity, pseudogap and quasiparticle dynamics. New J. Phys. 12, 105008 (2010).

2. Timusk, T. \& Statt, B. The pseudogap in high-temperature superconductors: an experimental survey. Rep. Prog. Phys. 62, 61-122 (1999).

3. Hüfner, S., Hossain, M. A., Damascelli, A. \& Sawatzky, G. A. Two gaps make a high-temperature superconductor? Rep. Prog. Phys. 71, 062501 (2008).

4. Littlewood, P. \& Kos, Ś. Focus on the Fermi surface. Nature 438, 435 (2005).

5. Mannella, N. et al. Nodal quasiparticle in pseudogapped colossal magnetoresistive manganites. Nature 438, 474-478 (2005).

6. Matthias, B. T. et al. Superconductivity and antiferromagnetism in Boron-rich lattices. Science 159, 530 (1968).

7. Lortz, R. et al. Specific heat, magnetic susceptibility, resistivity and thermal expansion of the superconductor $\mathrm{ZrB}_{12}$. Phys. Rev. B 72, 024547 (2005).

8. Wang, Y. et al. Specific heat and magnetization of a $\mathrm{ZrB}_{12}$ single crystal: Characterization of a type-II/1 superconductor. Phys. Rev. B 72, 024548 (2005).

9. Daghero, D. et al. Andreev-reflection spectroscopy in $\mathrm{ZrB}_{12}$ single crystals. Supercond. Sci. Technol. 17, S250-S254 (2004).

10. Gasparov, V. A., Sidorov, N. S. \& Zverkova, I. I. Two-gap superconductivity in $\mathrm{ZrB}_{12}$ : Temperature dependence of critical magnetic fields in single crystals. Phys. Rev. B 73, 094510 (2006)

11. Biswas, P. K. Studies of Unconventional Superconductors. Ph. D. Thesis (University of Warwick, Warwick, 2012).

12. Thakur, S. et al. Surface bulk differences in a conventional superconductor, $\mathrm{ZrB}_{12}$. J. Appl. Phys. 114, 053904 (2013).

13. Tsindlekht, M. I. et al. Tunneling and magnetic characteristics of superconducting $\mathrm{ZrB}_{12}$ single crystals. Phys. Rev. B 69, 212508 (2004).

14. Khasanov, R. et al. Anomalous electron-phonon coupling probed on the surface of superconductor $\mathrm{ZrB}_{12}$. Phys. Rev. B 72, 224509 (2005).

15. Leviev, G. I. Low-frequency response in the surface superconducting state of single-crystal ZrB 12 . Phys. Rev. B 71, 064506 (2005)

16. Yeh, J. J. \& Lindau, I. Atomic subsheell photoionization cross sections and asymmetry parameters: $1 \leq Z \leq 103$. At. Data and Nucl. Data Tables 32, 1 (1985).

17. Maiti, K. \& Singh, R. S. Evidence against strong correlation in $4 d$ transition-metal oxides $\mathrm{CaRuO}_{3}$ and $\mathrm{SrRuO}_{3}$. Phys. Rev. B 71, 161102(R) (2005).

18. Efros, A. F. \& Shklovskii, B. I. Coulomb gap and low temperature conductivity of disordered systems. I. Phys. C: Solid State Phys. 8, L49-L51 (1975).

19. Massey, J. G. \& Lee, M. Direct observation of the Coulomb correlation gap in a nonmetallic semiconductor, Si: B. Phys. Rev. Lett. 75, 4266-4269 (1995).

20. Altshuler, B. L. \& Aronov, A. G. Zero bias anomaly in tunnel resistance and electron-electron interaction. Solid State Commun. 30, 115-117 (1979).

21. Sarma, D. D. et al. Disorder effects in electronic structure of substituted transition metal compounds. Phys. Rev. Lett. 80, 4004-4007 (1998).
22. Kobayashi, M., Tanaka, K., Fujimori, A., Ray, S. \& Sarma, D. D. Critical test for Altshuler-Aronov Theory: Evolution of the density of states singularity in double perovskite $\mathrm{Sr}_{2} \mathrm{FeMoO}_{6}$ with controlled disorder. Phys. Rev. Lett. 98, 246401 (2007).

23. Medicherla, V. R. R., Patil, S., Singh, R. S. \& Maiti, K. Origin of ground state anomaly in $\mathrm{LaB}_{6}$ at low temperatures. Appl. Phys. Lett. 90, 062507 (2007).

24. Norman, M. R., Randeria, M., Ding, H. \& Campuzano, J. C. Phenomenology of the low-energy spectral function in high- $\mathrm{T}_{c}$ superconductors. Phys. Rev. B 57, R11093-R11097 (1998).

25. Shein, I. \& Ivanovskii, A. Band structure of superconducting dodecaborides $\mathrm{YB}_{12}$ and $\mathrm{ZrB}_{12}$. Phys. Solid State 45, 1429-1434 (2003).

26. Teyssier, J. et al. Optical study of electronic structure and electron-phonon coupling in $\mathrm{ZrB}_{12}$. Phys. Rev. B 75, 134503 (2007).

27. Maiti, K. Role of covalency in the ground-state properties of perovskite ruthenates: A first-principles study using local spin density approximations. Phys. Rev. B 73, 235110 (2006).

28. Singh, R. S. \& Maiti, K. Manifestation of screening effects and A-O covalency in the core level spectra of A site elements in the $\mathrm{ABO}_{3}$ structure of $\mathrm{Ca}_{1-x} \mathrm{Sr}_{x} \mathrm{RuO}_{3}$. Phys. Rev. B 76, 085102 (2007).

29. Lifshitz, I. M. Anomalies of electron characteristics of a metal in the high pressure region. Sov. Phys. JETP 11, 1130-1135 (1960).

30. Liu, C. et al. Evidence for a Lifshitz transition in electron-doped iron arsenic superconductors at the onset of superconductivity. Nat. Phys. 6, 419-423 (2010).

31. Balakrishnan, G., Lees, M. R. \& Paul, D. M. K. Growth of large single crystals of rare earth hexaborides. J. Crystal Growth 256, 206-209 (2003).

32. Blaha, P., Schwarz, K., Madsen, G. K. H., Kvasnicka, D. \& Luitz, J. WIEN2k An Augmented Plane Wave + Local Orbitals Program for Calculating Crystal Properties. Schwarz, K. (ed.) (Techn. Universität, Wien, 2001).

33. Paderno, Yu B., Liashchenko, A. B., Filippov, V. B. \& Dukhnenko, A. V. Advantages and Challenges, Science for Materials in the Frontier of Centuries: Skorokhod, V. V. (ed.) 347-348 (Kiev: IPMS, 2002).

34. Anisimov, V. I., Solovyev, I. V., Korotin, M. A., Czyzyk, M. T. \& Sawatzky, G. A. Density-functional theory and $\mathrm{NiO}$ photoemission spectra. Phys. Rev. B 48, 16929-16934 (1993).

\section{Acknowledgments}

The authors, K. M. and N. S. acknowledge financial support from the Department of Science and Technology under the Swarnajayanti Fellowship Programme. One of the author, G. B. wishes to acknowledge financial support from EPSRC, UK (EP/I007210/1).

\section{Author contributions}

S.T. carried out the measurements, band structure calculations, data analysis, and helped in manuscript preparation. D.B. and N.S. carried out photoemission measurements. P.K.B. and G.B. prepared the sample and characterized it. K.M. initiated the study, supervised the measurements, helped in data analysis, prepared the figures \& the manuscript.

\section{Additional information}

Competing financial interests: The authors declare no competing financial interests.

How to cite this article: Thakur, S. et al. Complex spectral evolution in a BCS superconductor, $\mathrm{ZrB}_{12}$. Sci. Rep. 3, 3342; DOI:10.1038/srep03342 (2013).

This work is licensed under a Creative Commons AttributionNonCommercial-NoDerivs 3.0 Unported license. To view a copy of this license, visit http://creativecommons.org/licenses/by-nc-nd/3.0 